\title{
Suppression of microRNA-9-5p rescues learning and memory in chronic cerebral hypoperfusion rats model
}

\author{
Na Wei ${ }^{1,2,3, *}$, Kai Zheng ${ }^{4, *}$, Rui Xue ${ }^{5}$, Sheng-Li Ma ${ }^{6}$, Hua-Yan Ren ${ }^{1,2,3}$, Hui-Fen \\ Huang $^{1,2,3}$, Wei-Wei Wang ${ }^{1,2,3}$, Jing-Jing $X u^{1,2,3}$ and Kui-Sheng Chen ${ }^{1,2,3}$ \\ ${ }^{1}$ Department of Pathology, The First Affiliated Hospital of Zhengzhou University, Zhengzhou 450002, People's Republic of \\ China \\ ${ }^{2}$ Henan Key Laboratory of Tumor Pathology, Zhengzhou 450002, People's Republic of China \\ ${ }^{3}$ Department of Pathology, School of Basic Medicine, Zhengzhou University, Zhengzhou 450002, People's Republic of China \\ ${ }^{4}$ Department of Geriatrics, Tongji Hospital, Tongji Medical College, Huazhong University of Science and Technology, Wuhan \\ 430030, China \\ ${ }^{5}$ Medical Research Center, The First Affiliated Hospital of Zhengzhou University, Zhengzhou 450002, People's Republic of \\ China \\ ${ }^{6}$ Department of Emergency, The First Affiliated Hospital of Zhengzhou University, Zhengzhou 450002, People's Republic of \\ China \\ *These authors have contributed equally to this work
}

Correspondence to: Jing-Jing XU, email: xujingjing_1982@163.com

Kui-Sheng Chen, email: kuishengchen@zzu.edu.cn

Keywords: miR-9-5p; memory; chronic cerebral hypoperfusion; long-term potentiation

Received: May 12, $2017 \quad$ Accepted: August 17, $2017 \quad$ Published: November 11, 2017

Copyright: Wei et al. This is an open-access article distributed under the terms of the Creative Commons Attribution License 3.0 (CC BY 3.0), which permits unrestricted use, distribution, and reproduction in any medium, provided the original author and source are credited.

\section{ABSTRACT}

Chronic cerebral hypoperfusion has been associated with cognitive impairment in dementias, such as Alzheimer's disease (AD) and vascular disease (VaD), the two most common neurodegenerative diseases in aged people. However, the effective therapeutic approaches for both $A D$ and $\mathrm{VaD}$ are still missing. MicroRNAs (miRNAs) are small non-coding RNAs that play important roles in the epigenetic regulation in many neurological disorders; the critical roles of miRNAderegulation had been implicated in both $A D$ and VaD. In the current study, we reported that miR-9-5p is elevated in the serum and cerebrospinalfluid of patientswith VaD. The miR-9-5p wasalso increased in both the hippocampus and cortex of rats with 2-vessel occlusionsurgery. Furthermore, application ofmiR-9-5p antagomirs attenuated the memory impairments in rats with 2-vessel occlusion surgery both in the Morris water maze and inhibitory avoidance step-down tasks. Furthermore, miR-9-5p antagomirs reducedthe inhibition oflongterm potentiation and loss of dendritic spines in chronic cerebral hypoperfusionrats. Additionally, the cholinergic neuronal function was rescued by miR-9-5p antagomirs, as well as the neuronal loss and the oxidative stress. We concluded that miR-9-5p inhibition may be a potential therapeutic target for the memory impairments caused by chronic cerebral hypoperfusion.

\section{INTRODUCTION}

Dementia is a broad category of brain diseases that cause a long term and gradualdecrement in emotionsand memory, which is great enough to reduce a person's ability to perform everyday activities [1]. There are severaltypes of dementia, including Alzheimer's disease (AD), vascular dementia (VaD), Lewy dody dementia (LBD), andfrontotemporal dementia (FTD) among others. Among them, $\mathrm{AD}$ and $\mathrm{VaD}$ are the two most prevalent 
types, which account for more than $80 \%$ of the dementia cases [2].

In the brain of $\mathrm{AD}$ and $\mathrm{VaD}$, the blood flow was reported to be reduced at an early stageand to be directly correlated with cognitive measures [3]. It is known that the flow of blood delivers the essential oxygen and nutrients to the brain cells and plays animportant rolein the maintenanceof normal thinking, learning, and memory [4]. The dramaticbloodflow reduction in patientswith $\mathrm{AD}$ orVaD may potentially lead to brain cell damage and cognitive decline $[5,6]$. Indeed, as a result of vascular risk factors such as hypertension, diabetes mellitus, hypercholesterolemia, and smoking, chronic cerebral hypoperfusion $(\mathrm{CCH})$ is a common vascular component among $\mathrm{AD}$ risk factors [7]. In $\mathrm{VaD}$, the deepbrain areas, particularly white matter areas, suffer from chronic and moderate ischemia, which is a state representing one of the physiopathological mechanisms of damage [8]. Consistent with this, the microvesselsin the $\mathrm{AD}$ and VaDbrainsare frequently narrowed, degenerated, and amyloid-laden, suggesting a pivotal role of cerebrovascularfactors in both $\mathrm{AD}$ and $\mathrm{VaD}[9$, 10]. Additionally, numerous studies have suggested that $\mathrm{CCH}$ might promote neurodegenerationthrough neuronal energy failure, production of reactive oxygen species, and proinflammatory cytokines by activated microglial cells that, in turn, damage the neuronal cells and contribute to white matter lesions [11].

MicroRNAs (miRNAs) are small (-22 nucleotides), endogenous noncoding RNA molecules that play important roles in diverse biological processes [12]. The mature miRNAis incorporated into the miRNA-induced silencing complex (miRISC) and then guides it to target sequences by recognizing their target sites located in 3'UTRs via incomplete base-pairing. The binding of miRNAwith its targets always leads tomRNAdestabilization or translational repression of the target genes $[13,14]$. The aberrant regulation of miRNAshad been reported in both $\mathrm{AD}$ and VaD brains $[15,16]$. Among them, miR-9, which is a highly conserved miRNAlocated on chromosome 3 in the mouse genome, is of particularinterest. Previous studies suggested that miR-9 is enriched in the brain, especially during development [17]. In AD brains, the level of miR-9 is increased in the temporal lobes, neocortex, and hippocampalregions when compared with age-matched healthy adults $[18,19]$. However, the role of miR-9 in the progression of memory impairment induced by vascular factors hasnot been studied yet.

In this study, we reported that miR-9-5p is upregulatedin both the serum and cerebrospinalfluid of patients with $\mathrm{VaD}$ and in the hippocampus of $\mathrm{CCH}$ rats. Furthermore, reduction of miR-9-5p by antagomirsrescued the learning and memory ability, synaptic plasticity, dendritic spines, cholinergic neurons, oxidative stress level, and neuronal loss induced by $\mathrm{CCH}$.

\section{RESULTS}

\section{miR-9-5p is upregulated in patients with VaD and CCHrats}

We first analyzed the miR-9-5p level in the serum and cerebrospinalfluid (CSF) of patients with $\mathrm{VaD}$ as described above. We found that in the serum of patientswith $\mathrm{VaD}$, the level of miR-9-5p increased to about 2.4 folds of age-matched controls (Figure 1A). A more prominent increment was found in the CSF samples (Figure 1B). As a miRNAcontrol, the level of miR-16
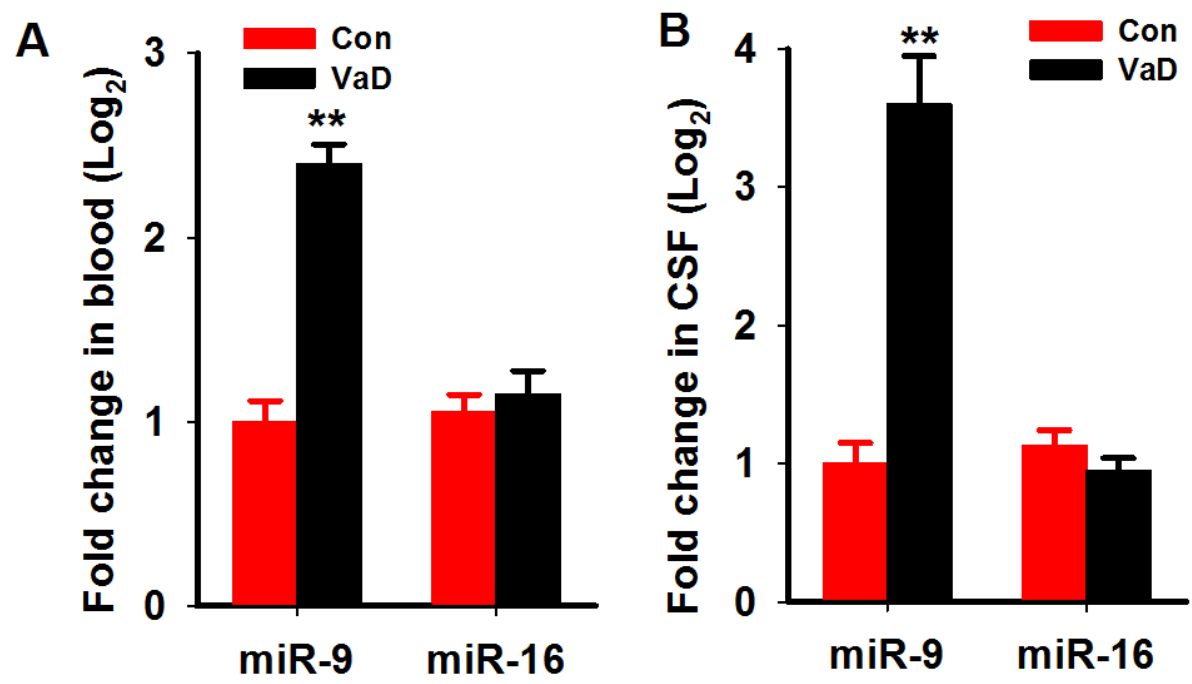

Figure 1: MiR-9-5p is upregulated in the serum and CSF of patientswith VaD. The serum and CSFof patients with VaD and age-matched controls were collected as described in Methods. The level of miR-9-5p and miR-16 in the serum (A) and CSF (B) were detected by Q-PCR. CSF, cerebrospinal fluid; VaD, vascular dementia; ${ }^{* *}, P<0.01$, compared with the controls. 
wasnot changed in boththe serum and CSF (Figure 1A, 1B). We further examined the miR-9-5p level in the hippocampi and corticestissues of rats at 3 monthafter the 2VO surgery. As expected, we detected an increase inmiR-9-5plevels both in the hippocampus andcortex ofthe $\mathrm{CCH}$ rats (Figure 2A, 2B). Similarly to our findings in patients, the levels of miR-16 werenot changed but the levels of miR-181c were reducedin the $\mathrm{CCH}$ rats (Figure $2 \mathrm{~A}, 2 \mathrm{~B})$. These data strongly suggested that miR-9-5p is upregulatedin patients with $\mathrm{VaD}$ and $\mathrm{CCH}$ rats.

\section{Reduction of miR-9-5p in vivo rescues learning and memory impairments in $\mathrm{CCH}$ rats}

To understand the effects of miR-9-5p increment in the pathogenesis of $\mathrm{VaD}$, we artificially suppressedthe expression of miR-9-5p in the brains of theCCH ratswith rno-miR-9-5p antagomirat 3 months after the surgery (Figure 3A). Subsequently, 2 weeks later, the spatial learning and memory abilities of the rats were evaluated with the MWM task. The swimmingtracks of the rats revealedthat the sham rats reached the platform inless than $20 \mathrm{~s}$ by using a direct searching strategy, while the $\mathrm{CCH}$ rats tookover $40 \mathrm{~s}$ using a random searching strategy. Treatingthe rats with anta-miR-9-5p improved the searching strategy (Figure 3B). On the first 2trainingdays, we did notdetectany significant difference among the groups. Beginning on training day 3, the escape latency of the $\mathrm{CCH}$ group was longer than that of the sham group, and treatment with anta-miR-9-5p significantly decreased the latencyin the $\mathrm{CCH}+$ Anta-miR-9-5pgroup comparedwith the $\mathrm{CCH}$ group (Figure $3 \mathrm{C}$ ). In the probe trial, rats in the $\mathrm{CCH}$ group displayed less crossing times to the platform region and less duration and distance in the target quadrantthan the sham group, while treatment with Anta-miR-9-5p improved thesemeasuresinCCH rats (Figure 3D-3F). No obvious difference was detectedbetween the Conand Anta-miR-9-5prats (Figure 3D-3F).

We used the step-down inhibitory avoidance test to evaluate the emotional cognition of the rats. During training, nosignificant differences were found in the step-down latency among the groups. In the test period, $\mathrm{CCH}$ rats showed obviously a shorter latency than control rats, whileanta-miR-9-5pextended the latency of $\mathrm{CCH}$ rats (Figure 4A). We also calculated the errors in both the training and test periods and found that $\mathrm{CCH}$ rats made more errors in both stages, while anta-miR-9-5p reduced the number of errors (Figure 4B). No significant differences were found between the Conand Anta-miR-9$5 p$ groups. The above results indicated that suppression of miR-9-5p is able to reverse the memory deficits in $\mathrm{CCH}$ rats.

\section{Reduction of miR-9-5p in vivo rescues the synaptic impairments in $\mathrm{CCH}$ rats}

Previous studies suggested that synaptic plasticity is the basis for learning and memory. We examined synaptic plasticity by evaluating LTP modificationsin the hippocampus of $\mathrm{CCH}$ rats. We found that the $\mathrm{CCH}$ rats displayed decreased slope of EPSP, which were lower than the ratsof the Sham group. Treatment withantamiR-9-5pelevated the declined LTP induced by $\mathrm{CCH}$
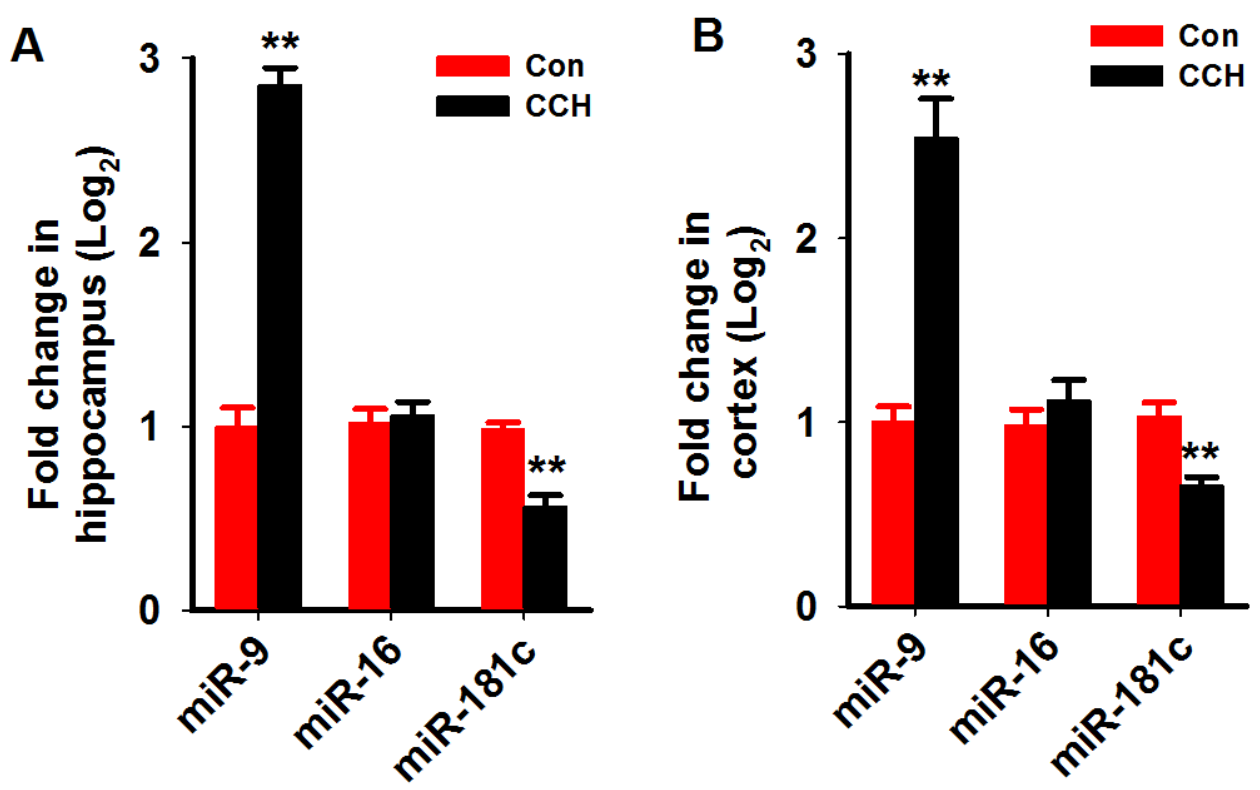

Figure 2: MiR-9-5p is increased in the hippocampus and cortex of CCH rats. Three months after the $2 \mathrm{VO}$ surgery, the hippocampus (A) and cortex (B) were extracted and the RNA wasisolated. The levels of miR-9-5p and miR-16 were evaluated by Q-PCR by using specific primers. 2VO, 2-vessel occlusion; $\mathrm{CCH}$, chronic cerebral hypoperfusion; ${ }^{* *}, P<0.01$, compared with Conrats. 

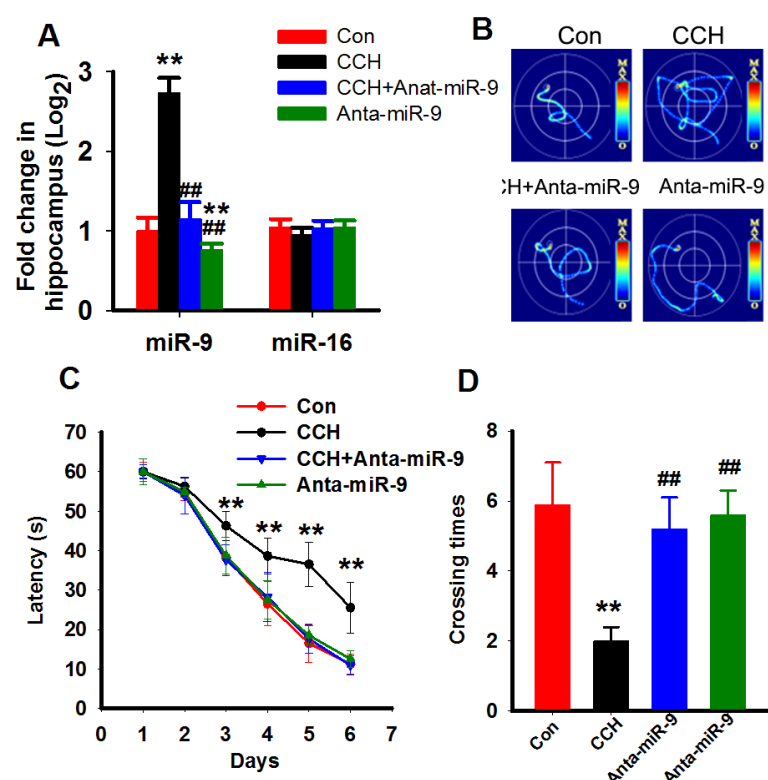

D
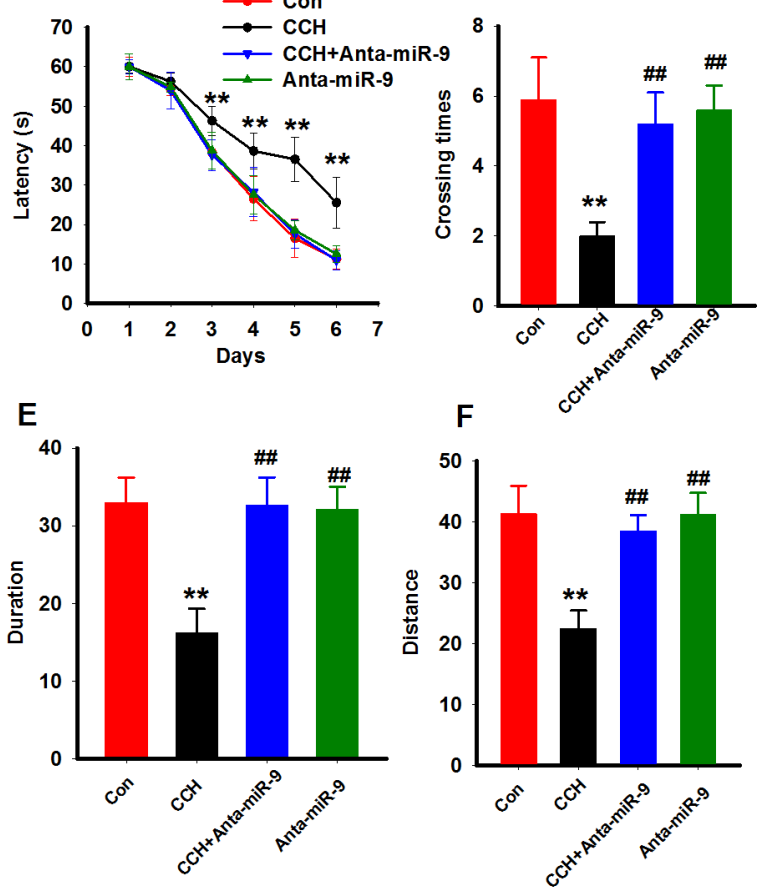

Figure 3: Inhibition of miR-9-5p rescued the spatial learning memory impairments of $\mathbf{C C H}$ rats. Three months after the surgery, Morris water maze was applied to evaluate the spatial memory of rats. (A) The miR-9-5p and miR-16 levels in different treated rats. ${ }^{* *} P<0.01$, compared to control rats. ${ }^{\#} P<0.01$, compared withCCH rats. (B) The representative escape traces for different groups in the final training day. (C) The latency during the six-days training task in the Morris water maze. (D-F) The total crossing times (D), the total time spent in the target quadrant (E), and the total swimming distances during the probe trial $(\mathbf{F})$. $\mathrm{CCH}$, chronic cerebral hypoperfusion; ${ }^{* *} P<0.01$, compared with sham rats; ${ }^{*} P<0.01$, compared with $\mathrm{CCH}$ rats.
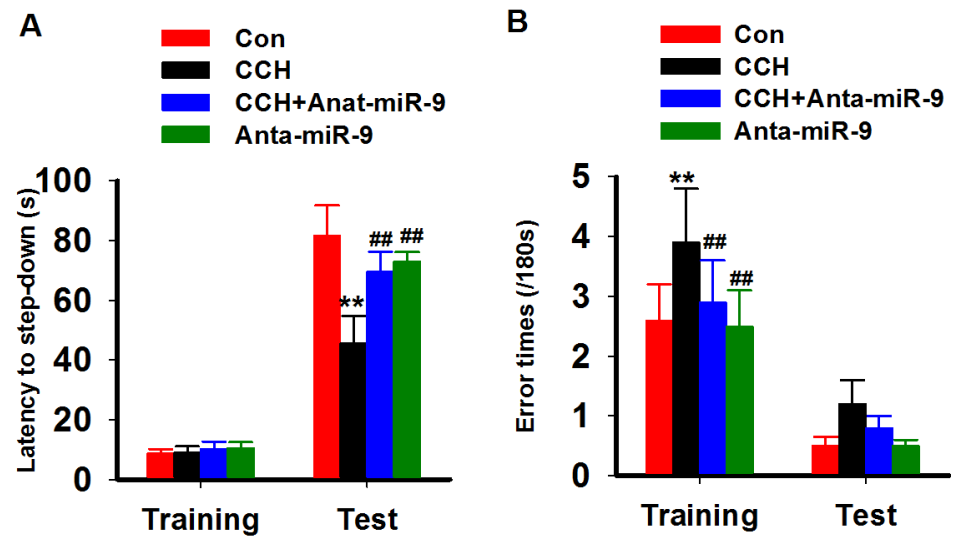

Figure 4: Inhibition of miR-9-5p rescued the fear memory impairments of $\mathrm{CCH}$ rats. The inhibitory avoidance tasks are applied to analyze fear memory of rats. After training, long-term memory test are carried at $24 \mathrm{~h}$ later. The step-down latency (A) and error times (B) were recorded. CCH, chronic cerebral hypoperfusion; ${ }^{* *} P<0.01$, compared with Conrats; ${ }^{* \#} P<0.01$, compared with CCH rats. 
(Figure 5A, 5B). No significant differences were found between the Conand Anta-miR-9-5p groups. Since dendritic spines are animportant morphological basis for synaptic plasticity, we also examined the dendritic spines in the dentate gyrususingGolgistaining. As expected, we found that $\mathrm{CCH}$ significantly decreased not only the density of dendritic spines but also the percentage of mushroom-type spines (Figure 6A-6C). The pre-administrated ofanta-miR-9-5p strongly improved theCCH-inducedspinogenesisinhibition. These results strongly implied that the in vivo suppression of miR-9-5p is able to reverse the synaptic impairments in $\mathrm{CCH}$ rats.

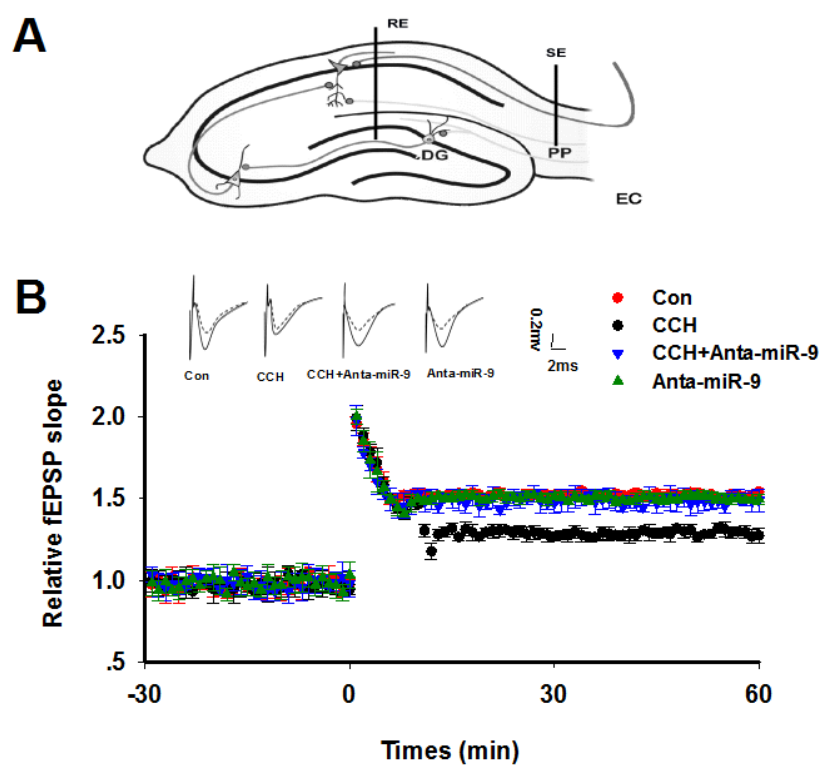

Figure 5: Inhibition of miR-9-5p rescued the LTP inhibition in CCH rats. (A) The diagram for LTP recording. RE: recording electrode; SE: stimulating electrode; PP: perforant path; DG: dentate gyrus. (B) Upper panel: The representative analog traces of evoked potentials before (dashline) and after (solid line) HFS with different treatmentsare shown. Lower panel: The alterations of LTP represented by normalized slope of EPSP were recorded. The electrophysiology recording was started 30 min (-30), and the HFS was added at 0 min. The data represent means \pm standard error of the mean. $\mathrm{CCH}$, chronic cerebral hypoperfusion; EPSP, excitatory postsynaptic potential; HFS, high frequency stimulation; LTP, long-term potentiation.

A

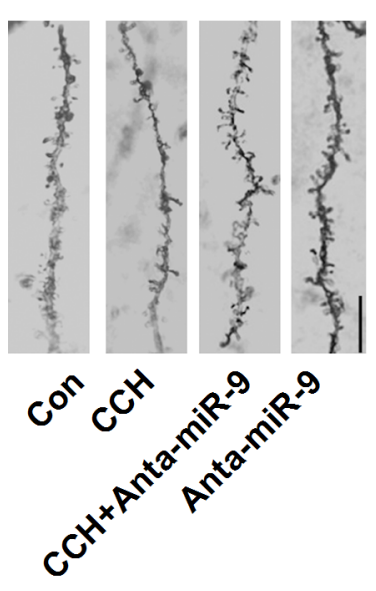

B

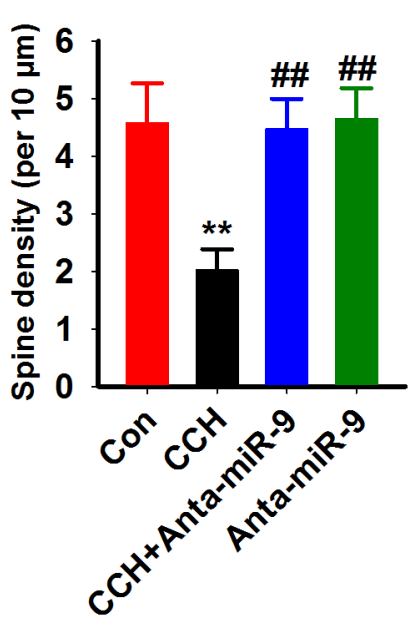

C

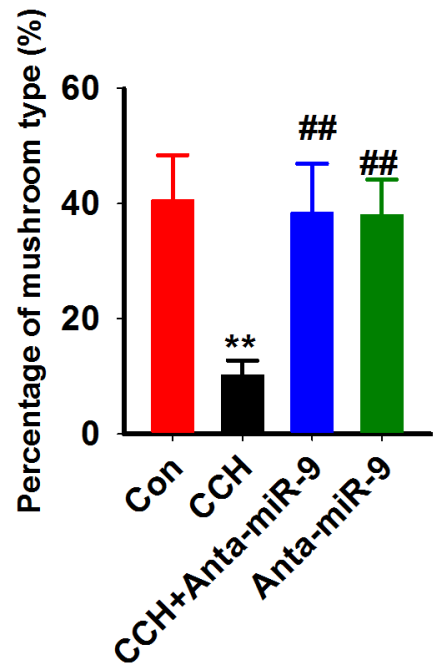

Figure 6: Inhibition of miR-9-5p rescued the dendritic spines abnormalities in CCH rats. (A) Representative images of dendritic spine were captured from Golgi staining of DG region. Bar $=20 \mu \mathrm{m}$. (B, C) The quantitative analysis of the density of spines (B) and the percentage of mushroom types (C) were carried. $\mathrm{CCH}$, chronic cerebral hypoperfusion; ${ }^{* *} P<0.01 v s . \mathrm{Congroup} ;{ }^{\# \#} P<0.01 v s$. $\mathrm{CCH}$ group. 


\section{Reduction of miR-9-5p in vivo reduces the cholinergic system in $\mathrm{CCH}$ rats}

Previous studies suggested that the central cholinergic system dysfunction is involved in memory impairments inCCH rats [20]. Therefore, we measured the effect of suppression of miR-9-5p on AChlevelsand activity of AChE and ChAT in the hippocampus of $\mathrm{CCH}$ rats. Consistent withprevious reports [21], $\mathrm{CCH}$ resulted in a significant decrease of $\mathrm{ACh}$ levels, increased $\mathrm{AChE}$ activity, and a dramatic reduction of ChAT activity in the hippocampus, which confirmed the impaired cholinergic function in $\mathrm{CCH}$. Moreover, we found that suppression of miR-9-5pby anta-miR-9-5p in $\mathrm{CCH}$ rats elevated AChlevelsand ChAT activity, butdecreased AChE activity. No significant differences were found between the Conand anta-miR-9-5p groups (Figure 7A-7C). These data support the behavioral results and suggest that reduction of miR$9-5 p$ may exert its neuroprotective effectsby restoring thecholinergic function.

\section{Reduction of miR-9-5p in vivo reduces the neuronal loss in $\mathrm{CCH}$ rats}

It was reported that neuronal loss resulting from apoptotic or necroticneuronal cell death is a common feature of $\mathrm{VaD}$ [22]. We also examined whether the reduction of miR-9-5p in vivo could reverse the neuronal loss in $\mathrm{CCH}$ rats by using the Nissl staining. Quantitative analysis suggested that $\mathrm{CCH}$ induces a dramatic neuronal loss in the hippocampuswhile pre-administrationofantamiR-9-5p strongly restored the number ofneurons in $\mathrm{CCH}$ rats (Figure 8A, 8B).

\section{Reduction of miR-9-5p inhibits the oxidative stress level in $\mathrm{CCH}$ rats}

As the oxidative stress was reported to induce neuronal loss in $\mathrm{CCH}$ rats [23], we examined the activities of SOD, GSH-px, and the levelsof MDA and T-ROS in the hippocampal homogenates of $\mathrm{CCH}$ rats. As predicted, the
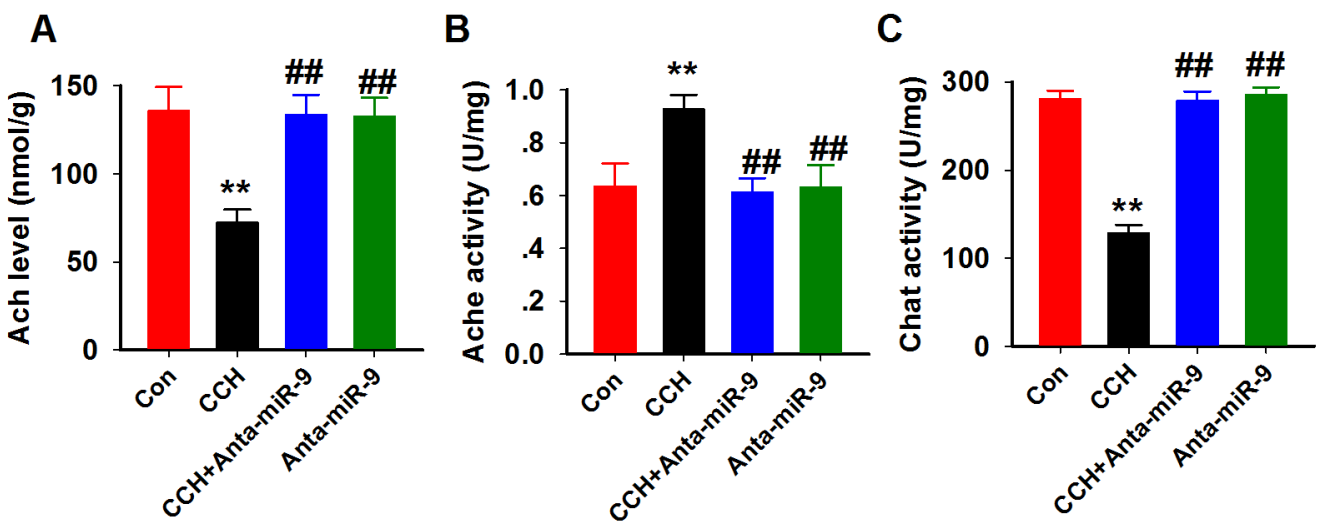

Figure 7: Inhibition of miR-9-5p rescued the cholinergic dysfunction in CCH rats. The cholinergic system function was assayed using ACh (A), AChE (B) and ChAT (C) kits as described above. CCH, chronic cerebral hypoperfusion; ${ }^{* *} P<0.01 v s$. Congroup; ${ }^{\#} P<0.01 v$ s. CCH group.

A

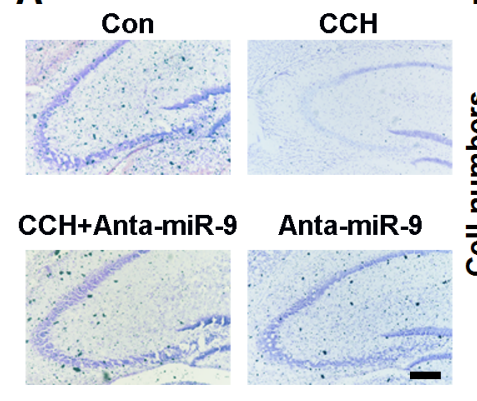

B

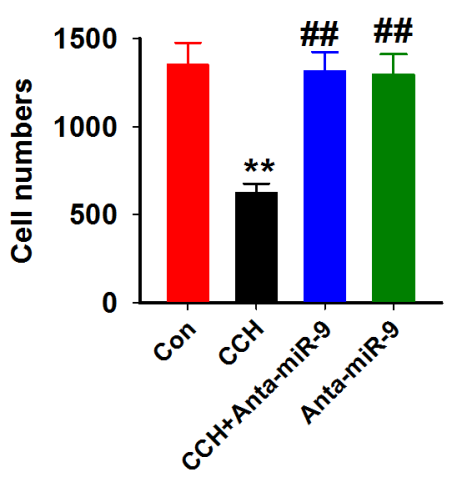

Figure 8: Inhibition of miR-9-5p restored the neuronal loss in CCH rats. (A) Arepresentative image ofthe hippocampus; scale bar $=100 \mu \mathrm{m}$. (B) The quantitative analysis for the whole hippocampus. $\mathrm{CCH}$, chronic cerebral hypoperfusion; ${ }^{* *} P<0.01 v s$. Congroup; ${ }^{\# \#} P<$ 0.01 vs. $\mathrm{CCH}$ group. $\mathrm{N}=16$ slices from 4 rats. 
SOD and GSH-px activities werereduced, but the levels of MDA and T-ROS were increased compared with controls. Administration of anta-miR-9-5p significantly restored the activities of SOD and GSH-pxand decreased the levels of MDA and T-ROS in the CCH rats (Figure 9A-9D). These data suggested that suppression of miR-9-5p inhibits the oxidative stress level in $\mathrm{CCH}$ rats.

\section{DISCUSSION}

$\mathrm{VaD}$ is thought to be a neurodegenerative disorder caused by multiple vasculotoxic and neurotoxic effects due to diminished cerebral blood flow, which leads to hypoxia and altered permeability of the blood-brain barrier. Severalpathological events have been suggested to be the prime risk factorsfor $\mathrm{VaD}$, such as stroke, cerebral hemorrhage, trauma, chronic diseases likeatherosclerosis, large and small vessel disease, and cardioembolic disease [24]. To date, the genetic basis of VaDisnot well defined. MiRNAs belong to a class of endogenous, stable, noncoding RNA molecules involved in the regulation of target gene expressionat the post-transcriptional levelby either the degradation of the RNA or translational arrest [25]. Several miRNAs are expressed specifically in the $\mathrm{CNS}$, where some are proposed to function in neuronal activities such as neurite outgrowth and synapse formation
[26], and in neurodegenerative diseases [27, 28], including $\mathrm{VaD}[15]$.

Our results demonstrated that miR-9-5p is critically involved in $\mathrm{VaD}$ and that blocking miR$9-5 p$ is sufficientto rescue the learning and memory impairments observed ina VaDrat model. The miR-9-5p gene is evolutionary well conserved. To date, several functional studies on miR-9 have emphasizeditsrole in neuronal development and neurogenesis [29]. For example, miR-9 knock-out mice, in which both miR-9-5p and $\mathrm{miR}-9-3 \mathrm{p}$ are reduced, displayed obvious defects in neurogenesisand abnormal telencephalicstructures [30]. It should be mentioned that miR-9-5p and miR-9-3p are abundantly expressed not only in neural progenitors but also in postmitotic neurons [17, 31]; hence the importance of studying the potential role of miR-9 in adulthood and neurological diseases. Indeed, in the postmortem brains of patients withHuntington's disease, the level of miR9 was decreased [32]. In brains from patients withAD, the level of miR-9wasincreased in the temporal lobes, neocortex, and hippocampalregions when compared withage-matched healthy adults $[18,19]$. In the current study, we demonstratedan abnormal upregulation of miR-9-5p in both the serum and CSF of patientswith $\mathrm{VaD}$, suggesting thepotential role of miR-9-5p in $\mathrm{VaD}$ progression.
A
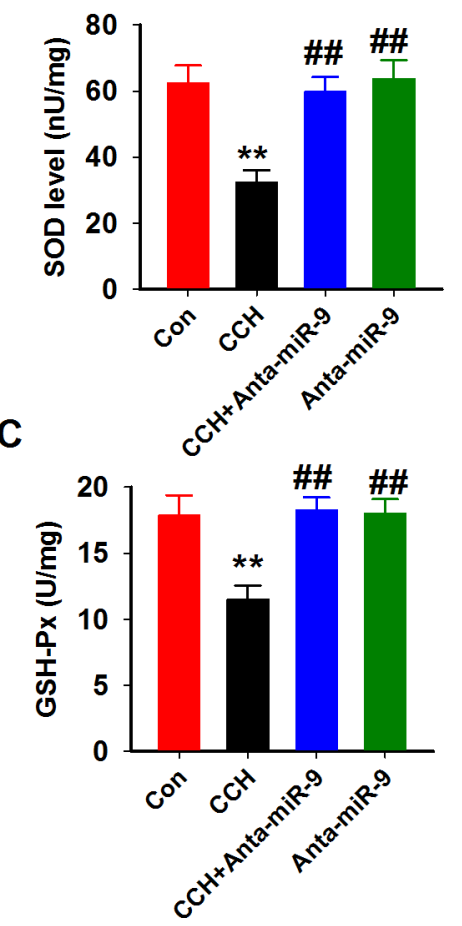

B
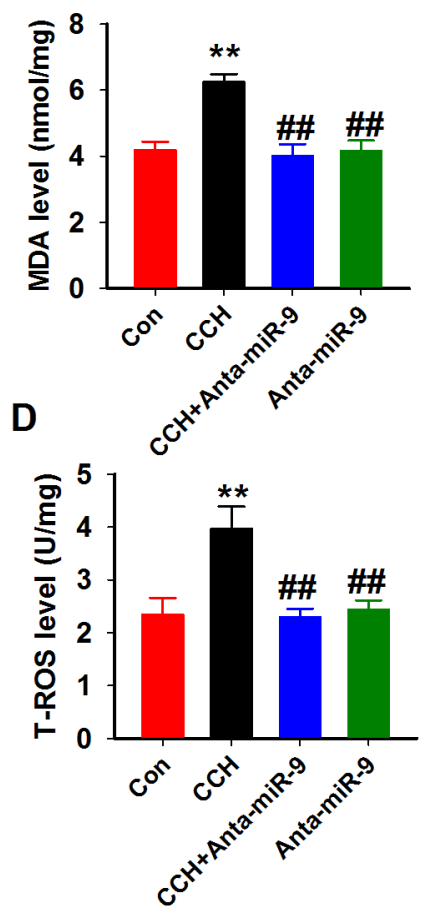

Figure 9: Inhibition of miR-9-5p attenuated the oxidative stress in CCH rats. Four oxidative stress parameters were assayed in the hippocampus homogenates from different groups by using SOD (A), MDA (B), GSH-Px (C) and T-ROS (D) kits described above. $\mathrm{CCH}$, chronic cerebral hypoperfusion; GSH-Px, glutathione peroxidase; MDA, malonic dialdehyde; SOD, superoxide dismutase; T-ROS, reactive oxygen species ${ }^{* *} P<0.01$ vs. control group; ${ }^{\#} P<0.01$ vs. $\mathrm{CCH}$ group. 
Synaptic plasticity and dendritic spines are the basis for learning and memory. Dendritic spines serve as a storage site for synaptic strength and help transmit electrical signals to the neuron's cell body [33]. Spines constitute a specialized compartment that contains multiple signaling complexes, which play important roles in synaptic transmission [34]. The disruption of synapses playsan important role in $\mathrm{VaD}$ [35]. Thus, the preservation of dendritic spines predictably restores synaptic plasticity, as well as learning and memory. Recently, in primary culture neurons, Giusti et al. [48] showed that the inhibition of miR9-5p using the sponge technique impairs dendritic growth and excitatory synaptic transmission. Interestingly, the miR9-3p sponge had no effect on dendritic growth of cultured neurons [36]. In an independent study, asynergistic effectof miR-9 and miR-124 has been reportedin the regulation of dendritic branching via the AKT/GSK3 $\beta$ pathway by targeting the Rap GTP-binding proteinRap2a [37]. In our study, we found that the administration of miR-9-5p antagomir restores the dendritic spines loss and the synaptic plasticity inhibition in the $\mathrm{CCH}$ rats. Moreover, miR-9-5p antagomir did not alter the dendritic spines and LTP in normal rats. These data suggested a discrepancy inthe in vivo and in vitro roles of miR-9-5p in dendritic spines and synaptic plasticity. We propose that such differences may be the result of the more complicated environmentin the brain compared with that of cultured neurons.

\section{MATERIALS AND METHODS}

\section{Patients and selection criteria}

A total of 25 patients with $\mathrm{VaD}$ (14 men and 11 women) whose diagnoses were confirmed using the National Institute of Neurological Disorders and Stroke-Association Internationale pour la Recherche et l'Enseignement en Neurosciences (NINDSAIREN) criteria for VaD [38]. The Mini-Mental State Examinationscores of all patients were less than 25. A total of 22 healthy age-matched participants (12 men and 10 women) were recruited from the outpatient setting and served as the control group. The control group was selected from the Medical Examination Centre of the First Affiliated Hospital of Zhengzhou University. In this study, none of the participants presented with hypertension, cardiopathy, diabetes, or renal dysfunction. All experiments using human samples were performed in accordance with the Declaration of Helsinki and the approval of the Institutional Review Board of Zhengzhou University.

\section{Animals and surgery}

Male Wistar rats (200-250 g) were obtained from the Animal Center of Zhengzhou University and were kept in standard plastic cages. During one week of acclimatization, rats were randomly distributed in pairs per cage. They were maintained on ad libitum food and water with 12/12 h light/dark cycle. All animal experiments were performed in accordance with the National Institutes of Health Guide for the Care and Use of Laboratory Animals and approved by the Animal Ethics Committee of the Medical School of Zhengzhou University.

After one week of acclimatization, rats were randomly divided into four groups, (1) control rats with the sham operation and vehicle injection (Con), (2) 2VO surgery and vehicle injection $(\mathrm{CCH}),(3) 2 \mathrm{VO}$ surgery with anta$m i R-9-5 p$ injection $(\mathrm{CCH}+$ Anta-miR-9-5p), and (4) antamiR-9-5p injection alone (Anta-miR-9-5p). For the 2VO surgery, rats were anesthetized with chloral hydrate (300 $\mathrm{mg} / \mathrm{kg}$, intraperitoneal [i.p.]), and bilateral common carotid arteries were gently separated from the carotid sheath and vagal nerves. Each artery was ligated with a 6-0 silk suture. Sham-operated controls received the same operation without ligation. After the procedure, rats were placed on a heating pad until recovery from anesthesia to maintain the body temperature at $37.5 \pm 0.5^{\circ} \mathrm{C}$ [23]. The antagomir of miR-9$5 \mathrm{p}(200 \mathrm{nM}$ in aCSF) was administered through stereotaxic brain injection to the DG area (AP -2.0 mm, ML $1.5 \mathrm{~mm}$, DV $2.0 \mathrm{~mm}$ ) once at 3 monthsafter the surgery. Sham-operated and controlled $2 \mathrm{VO}$ animals received the same volume of scrambled control or vehicle. The mortality was $1 / 17(5.9 \%)$ in the Con group, 3/19 (15.8\%) in the CCH group, 2/15 (13.3 $\%$ ) in the $\mathrm{CCH}+$ Anta-miR-9 group, and 1/14 (7.1\%) in the Anta-miR-9 group. Mortality rates did not differ significantly among the four groups.

\section{RNA isolation and miRNA detection}

Total RNA was extracted from the cells and hippocampi using the Trizol Reagent (Invitrogen) according to the manufacturer's instructions. Real-time PCR reactions were performed using an ABI 7500 real-time PCR system (Applied Biosystems, CA, USA). Reverse transcription of the extracted miRNA was performed with miRNAspecific primers using the miRcute miRNA first-strand cDNA synthesis kit, and real-time PCR of miRNAs was performed using the miRcute miRNA qPCR detection kit according to the manufacturer's protocol (TIANGEN BIOTECH, China). U6 was used as endogenous controls and non-neoplastic brain tissues were used for calibration. The primers for detection of human (miRQ0000441-1-1) and rat (miRQ0000142-1-1) miR-9-5pwere provided by RiboBio Co., Ltd. (Guangzhou, China) and the primer for U6 are followed: sense: 5'-CTCGCTTCGGCAGCACA; anti-sense: 5'-AACGCTTCACGAATTTGCGT).

\section{Morris water maze}

The Morris water maze (MWM) task was carried out at 3 months post-surgery [39, 40]. The maze consisted of a circular water tank $(120 \mathrm{~cm}$ in diameter and 60 
$\mathrm{cm}$ in depth) that was filled to a depth of $32 \mathrm{~cm}$ with opaque water and maintained at $22-26{ }^{\circ} \mathrm{C}$. A hidden circular platform $(10 \mathrm{~cm}$ in diameter) was submerged approximately $1.5 \mathrm{~cm}$ below the surface of the water and was kept at the same location in the southwest quadrant throughout the training period. All rats (including the sham-operated group) were subjected to daily MWM tests after completing a visual acuity test, as described previously [41]. Spatial training of the hidden platform in the water maze was performed for 6 consecutive days. Each rat received two trials per day with an intertrial interval of $1 \mathrm{~min}$. The starting position (east, west, south, or north) for each trial was pseudo-randomly chosen and counterbalanced across all experimental groups. The swimming paths of the rats were monitored by a video camera linked to a computer. For each training trial, the latency to escape onto the hidden platform was recorded. The rats were given a maximum of $60 \mathrm{~s}$ to find the hidden platform. If the rat failed to find the platform within $60 \mathrm{~s}$, the training was terminated and a maximum score of $60 \mathrm{~s}$ was assigned. Each rat was placed on the platform for $30 \mathrm{~s}$ before being removed from the water maze. For the probe trial on the 9th day of training [42], rats were subjected to a single 60-s swim without a hidden platform during the whole task, and the following durations were recorded: (1) time spent in the target quadrant where the platform had been placed during training, (2) the crossing times to the platform region, and (3) the initial time to cross the platform region. Swimming speed was also analyzed to evaluate motor ability [43].

\section{Step-down inhibitory avoidance task}

Animals were subjected to training and test sessions in a step-down inhibitory avoidance task with an interval of $24 \mathrm{~h}$. This task involves learning not to stepdown from a platform in order to avoid a mild foot shock. The inhibitory avoidance apparatus was an acrylic box $(30 \mathrm{~cm} \times 30 \mathrm{~cm}$ $\times 30 \mathrm{~cm}$ ) with a floor consisting of parallel stainless steel bars (5 mm diameter) spaced $1 \mathrm{~cm}$ apart. A platform (5$\mathrm{cm}$ wide $\times 5-\mathrm{cm}$ high) was placed at one corner on the floor. Rats were placed on the platform and the time latency to step-down on the grid with all four paws was measured with an automatic device. During the training sessions, immediately after stepping down on the grid, the animals received an electrical foot shock $(0.4 \mathrm{~mA}, 1.0 \mathrm{~s}$ scrambled). During the test sessions, no foot shock was delivered and step-down latency (with a ceiling of $180 \mathrm{~s}$ ) was used as a measure of memory retention, as described in previous reports. The test sessions were performed 24 $\mathrm{h}$ after training to evaluate long-term memory [44, 45].

\section{Long-term potentiation recording}

The long-term potentiation (LTP) recording and assay methods were performed as previously described
[46]. Briefly, animals were anesthetized with chloral hydrate $(300 \mathrm{mg} / \mathrm{kg}$, i.p.) and placed on a stereotaxic instrument. The stimulating electrode was placed in the perforant path (anterior-posterior [AP] $-7.0 \mathrm{~mm}$, mediolateral [ML] 4.5mm, dorsoventral [DV] $3.5 \mathrm{~mm}$ ) and the recording electrode in the dentate gyrus region (AP $-2.0 \mathrm{~mm}$, ML $1.5 \mathrm{~mm}$, DV $2.0 \mathrm{~mm}$ ) of the hippocampus. The initial baseline responses were obtained by delivering a single pulse of stimulation once every $10 \mathrm{~s}$. For each recording experiment, a stable baseline for at least 30 min was required before the application of conditioning stimuli. LTP was elicited using high-frequency stimulation (HFS) consisting of four trains of 50 pulses delivered at $200 \mathrm{~Hz}$ with a $2 \mathrm{~s}$ intertrain interval. The slope of the excitatory postsynaptic potential (EPSP) was recorded for $60 \mathrm{~min}$ and calculations were done by a computerized program (RM6240BD; Chengdu, China).

\section{Golgi staining}

Golgi staining was performed as previously described [47]. Briefly, tissue slices ( 4-mm thick) were placed in a solution containing $5 \%$ chloral hydrate, $5 \%$ potassium dichromate, $10 \%$ formalin in $\mathrm{ddH}_{2} \mathrm{O}$ for 3 days and then subjected to $1 \%$ silver nitrate under continuous vacuum for 4 days. Subsequently, brain tissues were cut into $40-\mu \mathrm{m}$ thick sections with a vibratome and analyzed using a light microscope (Olympus BX60, Tokyo, Japan) with a 100X objective lens. The number of dendritic spines and the percent of mushroom-like spines on hippocampal DG pyramidal neurons were analyzed blindly (i.e. without knowledge of the treatment groups). For each experimental group, a minimum of 40 cells per animal (n $=4$ ) were analyzedbyImage-Pro Plus 6.0 software. The criteria for the spine analysis were defined according to a previous study [48].

\section{ELISA}

Twenty-four hours after the behavioral testing, rats were sacrificed under deep anesthesia. The hippocampi were subsequently dissected on ice and stored at -80 ${ }^{\circ} \mathrm{C}$ for further biochemical assays. The hippocampal samples were prepared as previously described [49]. For the analysis of the cholinergic function, the activity of choline acetyltransferase (ChAT) and acetylcholinesterase (AChE) was measured spectrophotometrically using commercial assay kits according to the manufacturer's instructions (Cat. No. A079-1, A024, Nanjing Jiancheng Biotech. Inc., Nanjing, China). The level of ACh in the hippocampalsupernatant was detected with an ELISA kit according to the manufacturer's instructions (Cat. No. A105-1, Nanjing Jiancheng Biotech. Inc., Nanjing, China). To measure oxidative stress, the activities of superoxide dismutase (SOD; Cat. No. A001-3), glutathione peroxidase (GSH-Px; Cat. No. A005), reactive oxygen 
species (T-ROS; Cat. No. E004), and the levels of malonic dialdehyde (MDA; Cat. No. A003-1) were examined using commercial kits (Nanjing Jiancheng Biotech., Inc., Nanjing, China).

\section{Nissl staining}

The rats were anesthetized with an i.p. overdose of chloral hydrate and then perfused transcardially with $0.9 \%$ Sodium Chloride at $4{ }^{\circ} \mathrm{C}$ followed by $4 \%$ paraformaldehyde in $0.1 \mathrm{M}$ phosphate-buffer (PB, pH 7.4). The whole brains were removed and postfixed in $4 \%$ paraformaldehyde at $4{ }^{\circ} \mathrm{C}$ for another $24 \mathrm{~h}$. After dehydration in $30 \%$ and $40 \%$ sucrose until sunk, the brains were rapidly frozen in isopentane and $25-\mu \mathrm{m}$ thick coronal sections were cut on a cryostat (CM 1950, Leica, Heidelberger, Germany). All the sections were used for Nissl staining, which was performed with $0.1 \%$ cresyl violet (Sigma, St Louis, MO, USA) to evaluate neuronal damage in the hippocampus. For cell counting, the NIH Image $\mathrm{J}$ software was used as previously reported [50].

\section{Statistical analysis}

All data were expressed as the mean \pm standard deviation (SD). Data were analyzed using two-way analysis of variance (ANOVA) followed by Duncan's multiple range test when appropriate. $P$ values less than $0.05(P<0.05)$ were considered statistically significant.

\section{Author contributions}

N.W., K.Z., J.J.X. and K.S.C initiated and organized the study. N.W.,K.Z., R.X., S.M., H.Y. R., H.F.H., W.W.W., performed the main experimental work. N.W. analysed the data and performed statistical analysis. N.W.,K.Z., J.J.X. and K.S.C wrote the manuscript. All authors approved the fnal version of the manuscript.

\section{CONFLICTS OF INTEREST} interests.

The authors declare that they have no competing

\section{FUNDING}

This work was supported by the National Natural Science Foundation of China (81600944, U1304804, 81570199), the Youth Innovation Fund of The First Affiliated Hospital of Zhengzhou University and the key research projects of HeNan higher education (17A310010,17A310029).

\section{REFERENCES}

1. Burns A, Iliffe S. Dementia. BMJ. 2009; 338:b75.
2. Battistin L, Cagnin A. Vascular cognitive disorder. A biological and clinical overview. Neurochem Res. 2010; 35:1933-1938.

3. Roher AE, Debbins JP, Malek-Ahmadi M, Chen K, Pipe JG, Maze S, Belden C, Maarouf CL, Thiyyagura P, Mo H, Hunter JM, Kokjohn TA, Walker DG, et al. Cerebral blood flow in Alzheimer's disease. Vasc Health Risk Manag. 2012; 8:599-611.

4. Hoyer S. Senile dementia and Alzheimer's disease. Brain blood flow and metabolism. Prog Neuropsychopharmacol Biol Psychiatry. 1986; 10:447-478.

5. de la Torre JC. Cerebrovascular and cardiovascular pathology in Alzheimer's disease. Int Rev Neurobiol. 2009; $84: 35-48$.

6. Gorelick PB, Scuteri A, Black SE, Decarli C, Greenberg SM, Iadecola C, Launer LJ, Laurent S, Lopez OL, Nyenhuis D, Petersen RC, Schneider JA, Tzourio C, et al. Vascular contributions to cognitive impairment and dementia: a statement for healthcare professionals from the american heart association/american stroke association. Stroke. 2011; 42:2672-2713.

7. de la Torre JC. Alzheimer disease as a vascular disorder: nosological evidence. Stroke. 2002; 33:1152-1162.

8. Pantoni L, Garcia JH. Pathogenesis of leukoaraiosis: a review. Stroke. 1997; 28:652-659.

9. Kalaria RN. Cerebral vessels in ageing and Alzheimer's disease. Pharmacol Ther. 1996; 72:193-214.

10. Ogata J. Vascular dementia: the role of changes in the vessels. Alzheimer Dis Assoc Disord. 1999; 13:S55-58.

11. Kitagawa K, Yagita Y, Sasaki T, Sugiura S, OmuraMatsuoka E, Mabuchi T, Matsushita K, Hori M. Chronic mild reduction of cerebral perfusion pressure induces ischemic tolerance in focal cerebral ischemia. Stroke. 2005; 36:2270-2274.

12. Bartel DP. MicroRNAs: genomics, biogenesis, mechanism, and function. Cell. 2004; 116:281-297.

13. He L, Hannon GJ. MicroRNAs: small RNAs with a big role in gene regulation. Nat Rev Genet. 2004; 5:522-531.

14. Bushati N, Cohen SM. microRNA functions. Annu Rev Cell Dev Biol. 2007; 23:175-205.

15. Ragusa M, Bosco P, Tamburello L, Barbagallo C, Condorelli AG, Tornitore M, Spada RS, Barbagallo D, Scalia M, Elia M, Di Pietro C, Purrello M. miRNAs plasma profiles in vascular dementia: biomolecular data and biomedical implications. Front Cell Neurosci. 2016; 10:51.

16. Femminella GD, Ferrara N, Rengo G. The emerging role of microRNAs in Alzheimer's disease. Front Physiol. 2015; 6:40.

17. Yuva-Aydemir Y, Simkin A, Gascon E, Gao FB. MicroRNA-9: functional evolution of a conserved small regulatory RNA. RNA Biol. 2011; 8:557-564.

18. Lukiw WJ. Micro-RNA speciation in fetal, adult and Alzheimer's disease hippocampus. Neuroreport. 2007; 18:297-300. 
19. Sethi P, Lukiw WJ. Micro-RNA abundance and stability in human brain: specific alterations in Alzheimer's disease temporal lobe neocortex. Neurosci Lett. 2009; 459:100-104.

20. Deiana S, Platt B, Riedel G. The cholinergic system and spatial learning. Behav Brain Res. 2011; 221:389-411.

21. Ni JW, Matsumoto K, Li HB, Murakami Y, Watanabe H. Neuronal damage and decrease of central acetylcholine level following permanent occlusion of bilateral common carotid arteries in rat. Brain Res. 1995; 673:290-296.

22. Xu L, Di Q, Zhang Y. Cell cycle proteins preceded neuronal death after chronic cerebral hypoperfusion in rats. Neurol Res. 2008; 30:932-939.

23. Saxena AK, Abdul-Majeed SS, Gurtu S, Mohamed WM. Investigation of redox status in chronic cerebral hypoperfusion-induced neurodegeneration in rats. Appl Transl Genom. 2015; 5:30-32.

24. Iadecola C. The pathobiology of vascular dementia. Neuron. 2013; 80:844-866.

25. De Smaele E, Ferretti E, Gulino A. MicroRNAs as biomarkers for CNS cancer and other disorders. Brain Res. 2010; 1338:100-111.

26. Delay C, Mandemakers W, Hebert SS. MicroRNAs in Alzheimer's disease. Neurobiol Dis. 2012; 46:285-290.

27. Eacker SM, Dawson TM, Dawson VL. Understanding microRNAs in neurodegeneration. Nat Rev Neurosci. 2009; 10:837-841.

28. Sonntag KC. MicroRNAs and deregulated gene expression networks in neurodegeneration. Brain Res. 2010; 1338:48-57.

29. Coolen M, Katz S, Bally-Cuif L. miR-9: a versatile regulator of neurogenesis. Front Cell Neurosci. 2013; 7:220.

30. Wheeler BM, Heimberg AM, Moy VN, Sperling EA, Holstein TW, Heber S, Peterson KJ. The deep evolution of metazoan microRNAs. Evol Dev. 2009; 11:50-68.

31. Liu DZ, Ander BP, Tian Y, Stamova B, Jickling GC, Davis $\mathrm{RR}$, Sharp FR. Integrated analysis of mRNA and microRNA expression in mature neurons, neural progenitor cells and neuroblastoma cells. Gene. 2012; 495:120-127.

32. Packer AN, Xing Y, Harper SQ, Jones L, Davidson BL. The bifunctional microRNA miR-9/miR-9* regulates REST and CoREST and is downregulated in Huntington's disease. J Neurosci. 2008; 28:14341-14346.

33. Bourne JN, Harris KM. Balancing structure and function at hippocampal dendritic spines. Ann Rev Neurosci. 2008; 31:47-67.

34. Kennedy MJ, Ehlers MD. Organelles and trafficking machinery for postsynaptic plasticity. Ann Rev Neurosci. 2006; 29:325-362.

35. Li H, Wang J, Wang P, Rao Y, Chen L. Resveratrol reverses the synaptic plasticity deficits in a chronic cerebral hypoperfusion rat model. J Stroke Cerebrovasc Dis. 2016; 25:122-128.
36. Giusti SA, Vogl AM, Brockmann MM, Vercelli CA, Rein ML, Trumbach D, Wurst W, Cazalla D, Stein V, Deussing JM, Refojo D. MicroRNA-9 controls dendritic development by targeting REST. Elife. 2014.

37. Xue Q, Yu C, Wang Y, Liu L, Zhang K, Fang C, Liu F, Bian G, Song B, Yang A, Ju G, Wang J. miR-9 and miR-124 synergistically affect regulation of dendritic branching via the AKT/GSK3beta pathway by targeting Rap2a. Sci Rep. 2016; 6:26781.

38. Roman GC, Tatemichi TK, Erkinjuntti T, Cummings JL, Masdeu JC, Garcia JH, Amaducci L, Orgogozo JM, Brun A, Hofman A, Moody DM, O'Brien MD, Yamaguchi T, et al. Vascular dementia: diagnostic criteria for research studies. Report of the NINDS-AIREN International Workshop. Neurology. 1993; 43:250-260.

39. Hai J, Su SH, Lin Q, Zhang L, Wan JF, Li H, Chen YY, Lu Y. Cognitive impairment and changes of neuronal plasticity in rats of chronic cerebral hypoperfusion associated with cerebral arteriovenous malformations. Acta Neurol Belg. 2010; 110:180-185.

40. Melani A, Cipriani S, Corti F, Pedata F. Effect of intravenous administration of dipyridamole in a rat model of chronic cerebral ischemia. Ann N Y Acad Sci. 2010; 1207:89-96.

41. Walsh CM, Booth V, Poe GR. Spatial and reversal learning in the Morris water maze are largely resistant to six hours of REM sleep deprivation following training. Learn Mem. 2011; 18:422-434.

42. Hu J, Huang HZ, Wang X, Xie AJ, Wang X, Liu D, Wang JZ, Zhu LQ. Activation of glycogen synthase kinase-3 mediates the olfactory deficit-induced hippocampal impairments. Mol Neurobiol. 2015; 52:1601-1617.

43. Peng Y, Xu S, Chen G, Wang L, Feng Y, Wang X. 1-3-nButylphthalide improves cognitive impairment induced by chronic cerebral hypoperfusion in rats. J Pharmacol Exp Ther. 2007; 321:902-910.

44. Lucena GM, Prediger RD, Silva MV, Santos SN, Silva JF, Santos AR, Azevedo MS, Ferreira VM. Ethanolic extract from bulbs of Cipura paludosa reduced long-lasting learning and memory deficits induced by prenatal methylmercury exposure in rats. Dev Cogn Neurosci. 2013; 3:1-10.

45. Zhou P, Chen Z, Zhao N, Liu D, Guo ZY, Tan L, Hu J, Wang Q, Wang JZ, Zhu LQ. Acetyl-L-carnitine attenuates homocysteine-induced Alzheimer-like histopathological and behavioral abnormalities. Rejuvenation Res. 2011; 14:669-679.

46. Wang SH, Liao XM, Liu D, Hu J, Yin YY, Wang JZ, Zhu LQ. NGF promotes long-term memory formation by activating poly (ADP-ribose) polymerase-1. Neuropharmacology. 2012; 63:1085-1092.

47. Wang $X$, Wang ZH, Wu YY, Tang H, Tan L, Wang X, Gao XY, Xiong YS, Liu D, Wang JZ, Zhu LQ. Melatonin attenuates scopolamine-induced memory/synaptic disorder 
by rescuing EPACs/miR-124/Egr1 pathway. Mol Neurobiol. 2013; 47:373-381.

48. Liu D, Tang H, Li XY, Deng MF, Wei N, Wang X, Zhou YF, Wang DQ, Fu P, Wang JZ, Hebert SS, Chen JG, Lu Y, Zhu LQ. Targeting the HDAC2/HNF-4A/miR-101b/AMPK pathway rescues tauopathy and dendritic abnormalities in Alzheimer's disease. Mol Ther. 2017; 25:752-764.
49. Wei N, Xiao L, Xue R, Zhang D, Zhou J, Ren H, Guo $\mathrm{S}$, Xu J. MicroRNA-9 mediates the cell apoptosis by targeting Bcl2111 in ischemic stroke. Mol Neurobiol. 2016; 53:6809-6817.

50. Miller DJ, Balaram P, Young NA, Kaas JH. Three counting methods agree on cell and neuron number in chimpanzee primary visual cortex. Front Neuroanat. 2014; 8:36. 